\title{
A study on convolutions in weighted lorentz spaces using steklov means
}

\author{
Ahmet Hamdi AVŞAR* \\ * Ballkesir University, Necatibey Faculty of Education, Balikesir. \\ Geliş Tarihi (Received Date): 14.07.2021 \\ Kabul Tarihi (Accepted Date): 17.11.2021
}

\begin{abstract}
In this study, some estimations of convolution type operators defined with the help of Steklov operator in weighted Lorentz space $L_{\omega}^{p, q}(\mathbb{T})$ are obtained. Also, some basic properties of convolution type operators in these spaces are investigated.
\end{abstract}

Keywords: Convolution, weighted lorentz spaces, fourier series, steklov means, approximation.

\section{Ağırlıklı lorentz uzaylarında steklov ortalaması yardımıyla konvolüsyonlar üzerine bir çalışma}

$\ddot{\mathbf{O} z}$

Bu çalışmada, ă̆ırlıklı Lorentz uzaylarında Steklov operatörü yardımıyla tanımlanan konvolüsyon tipli operatörlerin bazı değerlendirmeleri elde edildi. Ayrıca, bu uzaylarda, konvolüsyon tipli operatörlerin bazı temel özellikleri incelendi.

Anahtar kelimeler: Konvolüsyon, ağırlıkl lorentz uzaylarl, fourier serisi, steklov ortalaması, yaklaşım.

\section{Introduction}

Lorentz space was firstly introduced by G. G. Lorentz in [1]. By means of the weight functions satisfying Muckenhoupt condition, the weighted Lorentz spaces were defined in $[2,3]$. We start with the definition and some properties of the weighted Lorentz space. As a first motivation for this, we define the weight function.

\footnotetext{
*Ahmet Hamdi AVŞAR, ahmet.avsar@balikesir.edu.tr, http://orcid.org/0000-0001-7277-1152
} 
Let $\mathbb{T}:=[-\pi, \pi]$ and the function $\omega: \mathbb{T} \rightarrow[0, \infty]$ be a measurable and nonnegative function. If the preimage set $\omega^{-1}(\{0, \infty\})$ has the Lebesgue measure zero then we say that $\omega$ is called a weight function. If $\eta$ is a measurable set, then we write

$\omega(\eta)=\int_{\eta} \omega(x) d x$

The decreasing rearrangement function $f_{\omega}^{*}(\tau)$ of $f: \mathbb{T} \rightarrow \mathbb{R}$ with respect to the Borel measure (1) is defined as

$f_{\omega}^{*}(\tau)=\inf \{\alpha \geq 0: \omega(x \in \mathbb{T}:|f(x)>\alpha|) \leq \tau\}$.

The average function $f^{* *}(\tau)$ is defined as follow

$f^{* *}(\tau)=\frac{1}{\tau} \int_{0}^{\tau} f^{*}(\vartheta) d \vartheta$

Let $f: \mathbb{T} \rightarrow \mathbb{R}$ be a measurable function. Assume that $1<p, q<\infty$. The weighted Lorentz space $L_{\omega}^{p, q}(\mathbb{T})$ consists of measurable functions defined [2, p. 219], [3, p. 20] as the set of all measurable functions $f$ for which

$\|f\|_{p q, \omega}=\left(\int_{\mathbb{T}}\left(f^{* *}(\tau)\right)^{q} \tau^{\frac{q}{p}} \frac{d \tau}{\tau}\right)^{\frac{1}{q}}<\infty$.

The weighted Lorentz space $L_{\omega}^{p, q}(\mathbb{T})$ is an extension of weighted Lebesgue space $L_{\omega}^{p}(\mathbb{T})$ [3, p. 20] since $L_{\omega}^{p, p}(\mathbb{T})=L_{\omega}^{p}(\mathbb{T})$. In this paper, we will use $L_{\omega}^{p, q}$ instead of $L_{\omega}^{p, q}(\mathbb{T})$.

The weights $\omega$ belongs to the Muckenhoupt class $A_{p}(\mathbb{T})$ [4] which is defined by the condition

$\sup \frac{1}{|J|} \int_{J} \omega(x) d x\left(\frac{1}{|J|} \int_{J} \omega^{1-p^{\prime}}(x) d x\right)^{p-1}<\infty, p^{\prime}=\frac{p}{p-1}$

where $J$ is any subintervals on $[0,2 \pi]$ and $|J|$ represents the lenght of $J$.

For $f \in L^{1}$, the Hardy Littlewood maximal function is defined as

$M(x ; f):=\sup _{x \in I} \frac{1}{|I|} \int_{I} f(\tau) d \tau, \quad x \in[0,2 \pi]$

where the supremum is taken over all subintervals $I$ of $[0,2 \pi]$ and $|I|$ represents the lenght of $I$. [5, p. 80].

When $\omega \in A_{p}(\mathbb{T}), 1<p, q<\infty$, the Hardy Littlewood maximal function of $f \in$ $L_{\omega}^{p, q}(\mathbb{T})$ is bounded in $L_{\omega}^{p, q}(\mathbb{T})[6]$.

Let $f, g \in L^{1}$. Then the convolution type operator is defined as

$(f * g)(x ; h):=\int_{0}^{2 \pi} \sigma_{h} f(x, u) g(u) d u$

where

$\sigma_{h} f(x, u):=\frac{1}{h} \int_{0}^{h} f(x+u t) d t, \quad 0<h<\pi, \quad x \in[0,2 \pi], \quad-\infty<u<\infty$

is the Steklov means constructed by means of $f$.

The basic properties of this type of convolution operator used in the structure of the approximation polynomials and modulus of smoothness were investigated in variable exponent Lebesgue space by Israfilov and Yirtici in [7] and weighted Lorentz spaces in 
[6,8-11]. In addition, properties of convolution type transforms were also examined in [12-15]. The properties of above type convolution may be established and investigated in weighted Lorentz spaces. In this study, we investigate the properties of the convolution type operators, and the approximation identities in weighted Lorentz spaces. Further information about this convolution can be found in [16].

\section{Main results}

We have the following two relations taking into account the definition given by (2) for $\forall K, L, M \in L_{\omega}^{p, q}$ and for $\forall \alpha \in \mathbb{R}$.

- $((\alpha K) * L)(x, h)=\alpha(K * L)(x, h)$,

- $((K \mp L) * M)(x, h)=(K * M)(x, h) \mp(L * M)(x, h)$.

Note: We note that this convolution type operator defined in terms of Steklov operator is not commutative. Example: Let $a, b \in \mathbb{R}, K$ be a constant function and $L$ be a linear function $(K(x)=a$ and $L(x)=a x+b)$. Then, we get

$$
\begin{aligned}
& (K * L)(x, h):=\int_{0}^{2 \pi}\left(\frac{1}{h} \int_{0}^{h} f(x+t u) d t\right) g(u) d u \\
& =\int_{0}^{2 \pi}\left(\frac{1}{h} \int_{0}^{h} a d t\right)(a u+b) d u \\
& =\int_{0}^{2 \pi} a(a u+b) d u \\
& =2 a \pi(a \pi+b) .
\end{aligned}
$$

At the same time,

$$
\begin{aligned}
& (L * K)(x, h):=\int_{0}^{2 \pi}\left(\frac{1}{h} \int_{0}^{h} g(x+t u) d t\right) f(u) d u \\
& =\int_{0}^{2 \pi}\left(\frac{1}{h} \int_{0}^{h}(a(x+t u)+b) d t\right) a d u \\
& =\int_{0}^{2 \pi}\left(a\left(x+\frac{u h}{2}\right)+b\right) a d u \\
& =a 2 \pi\left(a x+b+\frac{a h \pi}{2}\right) .
\end{aligned}
$$

We shall use following auxiliary result for proving the our main theorems.

Theorem 1 [10] Let $\omega \in A_{p}(\mathbb{T}), 1<p, q<\infty$ and $\xi$ be a measurable function of two variables. Then

$\left\|\int_{\mathbb{T}} \xi(x, \cdot) d x\right\|_{p q, \omega} \leq c \int_{\mathbb{T}}\|\xi(x, \cdot)\|_{p q, \omega} d x, c$ is a positive constant. 
Our main results are followings.

Theorem 2 Let $\omega \in A_{p}(\mathbb{T}), 1<p, q<\infty, f \in L_{\omega}^{p, q}(\mathbb{T})$ and $g \in L^{1}$. Then, there is a positive constant c such that $\|f * g\|_{p q, \omega} \leq c\|f\|_{p q, \omega}\|g\|_{L^{1}}$.

Theorem 3 Let $\omega \in A_{p}(\mathbb{T}), 1<p, q<\infty, f \in L_{\omega}^{p, q}(\mathbb{T})$ and $g \in L^{1}$. Then the convolution type transform $f * g$ can be approached in $L_{\omega}^{p, q}(\mathbb{T})$ by the finite linear combinations of means $f$, that is, for $\forall \epsilon>0$, there are the sets of numbers $\left\{\lambda_{k}\right\}_{1}^{n} \subset \mathbb{R}$ and $\left\{u_{k}\right\}_{1}^{n} \subset[0,2 \pi]$ such that

$$
\left\|(f * g)(\cdot, h)-\sum_{k=1}^{n} \lambda_{k} \sigma_{h} f\left(\cdot, u_{k}\right)\right\|_{p q, \omega}<\epsilon .
$$

Now we consider the identities of convergence in weighted Lorentz space $L_{\omega}^{p, q}(\mathbb{T})$. By the identity of convergence we understand a sequence $\left\{K_{n}\right\}_{n \in \mathbb{N}} \in L^{1}$ satisfying the following conditions:

a. $\sup \left\|K_{n}\right\|_{L^{1}}<\infty$;

b. $\lim _{n \rightarrow \infty} \frac{1}{2 \pi} \int_{-\pi}^{\pi} K_{n}(x) d x=1$;

c. $\lim _{n \rightarrow \infty} \frac{1}{2 \pi} \int_{\delta \leq|x| \leq \pi}\left|K_{n}(x)\right| d x=0, \forall \delta \in(0, \pi)$.

Under given conditions the following theorem is true.

Theorem 4 Let $\omega \in A_{p}(\mathbb{T}),\left\{K_{n}\right\}_{n \in \mathbb{N}}$ be an identity of convergence and $1<p, q<\infty$. Then for every $f \in L_{\omega}^{p, q}(\mathbb{T})$, the relation

$\lim _{n \rightarrow \infty}\left\|\frac{f * K_{n}}{2 \pi}-f\right\|_{p q, \omega}=0$

holds.

\section{Proof of main results}

Proof of Theorem 2 Let $f \in L_{\omega}^{p, q}(\mathbb{T})$. Using Theorem 1 and the boundedness of Hardy Littlewood maximal function we get

$\|(f * g)\|_{p q, \omega}=\left\|\int_{0}^{2 \pi} \sigma_{h} f(\cdot, u) g(u) d u\right\|_{p q, \omega}$

$\leq c \int_{0}^{2 \pi}\left\|\sigma_{h} f(\cdot, u)\right\|_{p q, \omega}\|g(u)\|_{p q, \omega} d u$

$\leq c\|f\|_{p q, \omega}\|g\|_{L^{1}}$.

Proof of Theorem 3 Let $S_{2 \pi}$ be the set of simple functions defined on $[0,2 \pi]$. Since $S_{2 \pi}$ is dense subset of $L^{1}$, it is sufficient to prove the Theorem 3 in the event of $g \in S_{2 \pi}$. Every function $g \in S_{2 \pi}$ can be expressed as a linear conjugation of the characteristic 
function of subintervals on $[0,2 \pi]$. Therefore it is sufficient to proof the Theorem 3 in the event of

$g(u):=\chi_{\varsigma}(u):=\left\{\begin{array}{ll}1, & u \in \varsigma \\ 0, & u \in \varsigma\end{array}\right.$,

where $\varsigma:[a, b], 0<a<b<2 \pi$, is a random interval.

For an arbitrary number $\delta>0$, we divide $\varsigma$ into a finite subintervals $I_{k}$ with length

$\left|I_{k}\right|<\delta$ and satisfying the conditions $I_{i} \cap I_{j}=\emptyset, i \neq j$ and $\varsigma=\cup_{k} I_{k}$. Then

$(f * g)(x, h):=\int_{0}^{2 \pi} \sigma_{h} f(x, u) g(u) d u$

$=\int_{0}^{2 \pi} \sigma_{h} f(x, u) \chi_{\varsigma}(u) d u$

$=\int_{0}^{2 \pi} \sigma_{h} f(x, u) d u$

$=\sum_{k} \int_{I_{k}}\left(\sigma_{h} f\right)(x, u) d u$.

Taking $u_{k} \in I_{k}$ we have

$$
\begin{aligned}
& (f * g)(x, h)-\sum_{k}\left|I_{k}\right|\left(\sigma_{h} f\right)\left(x, u_{k}\right) \\
& =\sum_{k} \int_{I_{k}}\left(\sigma_{h} f\right)(x, u) d u-\sum_{k} \int_{I_{k}}\left(\sigma_{h} f\right)\left(x, u_{k}\right) d u \\
& =\sum_{k} \int_{I_{k}}\left[\left(\sigma_{h} f\right)(x, u)-\left(\sigma_{h} f\right)\left(x, u_{k}\right)\right] d u .
\end{aligned}
$$

Considering the triangle property in $L_{\omega}^{p, q}(\mathbb{T})$ norm and Theorem 1 together, we have

$$
\begin{aligned}
& \left\|(f * g)(x, h)-\sum_{k}\left|I_{k}\right|\left(\sigma_{h} f\right)\left(x, u_{k}\right)\right\|_{p q, \omega}\|\|_{p q, \omega} \\
& =\left\|\sum_{k} \int_{I_{k}}\left[\left(\sigma_{h} f\right)(x, u)-\left(\sigma_{h} f\right)\left(x, u_{k}\right)\right] d u\right\|_{k} \\
& \leq c \sum_{I_{k}}\left\|\left[\left(\sigma_{h} f\right)(x, u)-\left(\sigma_{h} f\right)\left(x, u_{k}\right)\right]\right\|_{p q, \omega} d u .
\end{aligned}
$$

By the continuity of $\sigma_{h} f$, for $\forall \epsilon>0$ there is a $\delta>0$ such that for every finite subintervals $I_{k} \subset$ s such that $\left|I_{k}\right|<\delta$ and $u \in I_{k}$ the inequality

$\left\|\left[\left(\sigma_{h} f\right)(x, u)-\left(\sigma_{h} f\right)\left(x, u_{k}\right)\right]\right\|_{p q, \omega}<\frac{\epsilon}{2 \pi c}$

holds. Hence by (3) and (4) we get

$\left\|(f * g)(x, h)-\sum_{k}\left|I_{k}\right|\left(\sigma_{h} f\right)\left(x, u_{k}\right)\right\|_{p q, \omega} \leq c \sum_{k} \int_{I_{k}} \epsilon d u=c \sum_{k}\left|I_{k}\right| \frac{\epsilon}{2 \pi c}$ 
$=c \sum_{k}|\varsigma| \frac{\epsilon}{2 \pi c} \leq \epsilon$,

where $|\varsigma|$ is the Lebesgue measure of $\varsigma$.

Proof of Theorem 4 Let $f$ be a continuous function on $[-\pi, \pi]$, so we write $f \in$ $C([-\pi, \pi])$. Using the triangle inequality we get

$$
\begin{aligned}
& \left\|\frac{f * K_{n}}{2 \pi}-f\right\|_{p q, \omega} \leq\left\|\frac{f * K_{n}}{2 \pi}-\frac{1}{2 \pi} \int_{0}^{2 \pi} f(\cdot) K_{n}(u) d u\right\|_{p q, \omega} \\
& +\left\|\frac{1}{2 \pi} \int_{0}^{2 \pi} f(\cdot) K_{n}(u) d u-f\right\|_{p q, \omega} .
\end{aligned}
$$

By rearranging the right side of (5), we get

$$
\begin{aligned}
& \left|\frac{f * K_{n}}{2 \pi}-\frac{1}{2 \pi} \int_{0}^{2 \pi} f(x) K_{n}(u) d u\right| \\
& =\left|\frac{1}{2 \pi} \int_{0}^{2 \pi}\left(\frac{1}{h} \int_{0}^{h} f(x+u t) d t\right) K_{n}(u) d u-\frac{1}{2 \pi} \int_{0}^{2 \pi} f(x) K_{n}(u) d u\right| \\
& =\left|\frac{1}{2 \pi} \int_{0}^{2 \pi}\left(\frac{1}{h} \int_{0}^{h}[f(x+u t)-f(x)] d t\right) K_{n}(u) d u\right| \\
& \leq \frac{1}{2 \pi} \int_{0}^{2 \pi}\left(\frac{1}{h} \int_{0}^{h}[f(x+u t)-f(x)] d t\right)\left|K_{n}(u)\right| d u .
\end{aligned}
$$

Let $\epsilon>0$. Due to $0 \leq t \leq h$, by the continuity of $f$ for a given $\epsilon$ there is a $\delta>0$ such that for $h<\delta$, the inequality $|f(x+u t)-f(x)|<\epsilon$ holds. Therefore, considering $(a)$ we get

$\left|\frac{f * K_{n}}{2 \pi}-\frac{1}{2 \pi} \int_{0}^{2 \pi} f(x) K_{n}(u) d u\right|$

$\leq \epsilon \frac{1}{2 \pi} \int_{0}^{2 \pi}\left|K_{n}(u)\right| d u \leq c \epsilon$.

Also, considering $(b)$ we get

$\lim _{n \rightarrow \infty} \frac{1}{2 \pi} \int_{0}^{2 \pi} f(x) K_{n}(u) d u=f(x), x \in[0,2 \pi]$

and therefore for a given $\epsilon>0$ there is a $n_{0} \in \mathbb{N}$ such that for every $n \geq n_{0}$

$\left\|\frac{1}{2 \pi} \int_{0}^{2 \pi} f(\cdot) K_{n}(u) d u-f\right\|_{p q, \omega} \leq \epsilon$.

Using (6) and (7) in (5) we have 
$\left\|\frac{f * K_{n}}{2 \pi}-f\right\|_{p q, \omega}<c \epsilon$.

Let $1<p, q<\infty, f \in L_{\omega}^{p, q}(\mathbb{T})$. Since $C([-\pi, \pi])$ is dense in $L_{\omega}^{p, q}[1]$. For every $\epsilon>0$ there is a function $g \in C([-\pi, \pi])$ such that

$\|f-g\|_{p q, \omega} \leq \epsilon$.

If we consider $(a)$ and Theorem 1 together, we get

$\left\|\frac{f * K_{n}}{2 \pi}-\frac{g * K_{n}}{2 \pi}\right\|_{p q, \omega}=\left\|\frac{(f-g) * K_{n}}{2 \pi}\right\|_{p q, \omega}$

$\leq \frac{c}{2 \pi}\|(f-g)\|_{p q, \omega}\left\|K_{n}\right\|_{1}$

$\leq M_{0} \epsilon$

where $M_{0}$ is a positive constant idependent of $n$.

Now, if we consider (10), (8) and (9) for every $n \geq n_{0}$, the we get

$\left\|\frac{f * K_{n}}{2 \pi}-f\right\|_{p q, \omega} \leq\left\|\frac{f * K_{n}}{2 \pi}-\frac{g * K_{n}}{2 \pi}\right\|_{p q, \omega}+\left\|\frac{g * K_{n}}{2 \pi}-g\right\|_{p q, \omega}+\|(f-g)\|_{p q, \omega}$

$\leq M_{0} \epsilon+c \epsilon+\epsilon=\epsilon\left(M_{0}+c+1\right)$.

\section{References}

[1] Lorentz, G. G., Some new functional spaces, Annals of Mathematics, 51, 37 55, (1950).

[2] Bennet, B., and Sharpley, R., Interpolation of operators, Academic Press, Boston MA, (1968).

[3] Genebashvili, I., Gogatishvili, A., Kokilashvili, V. M. and Krbec, M., Weight theory for integral transforms on spaces of homogeneous type, 92, CRC Press, USA, (1997).

[4] Muckenhoupt, B., Weighted Norm Inequalities for the Hardy Maximal Function, Transactions of the American Mathematical Society, 165, 207-226, (1972).

[5] Cruz-Uribe, D.V. and Fiorenza, A., Variable Lebesgue spaces: Foundations and Harmonic Analysis, Springer Science-Business Media, (2013).

[6] Chang, H. M., Hunt, R. A. and Kurtz, D. S., The Hardy-Littlewood maximal functions on $\mathrm{L}(\mathrm{p} ; \mathrm{q})$ spaces with weights, Indiana University Mathematics Journal, 31, 109-120, (1982).

[7] Israfilov, D. M., and Yırtıc1, E., On some properties of convolutions in variable exponent Lebesgue spaces, Complex Analysis and Operator Theory, 11 (8), 1817-1824, (2017).

[8] Akgün R., Yildirir Y. E., Jackson-Stechkin type inequalities in weighted Lorentz spaces, Mathematical Inequalities and Applications, 18 (4), 1283.1293, (2015).

[9] Kokilashvili, V. M. and Krbec, M., Weighted inequalities in Lorentz and Orlicz spaces, World Scientific Publishing, (1991).

[10] Kokilashvili, V.M., and Yıldırır, Y. E., On the approximation by trigonometric polynomials in weighted Lorentz spaces, Journal of Function Spaces and Applications, 8 (1), 67-86, (2010). 
[11] Akgün, R., and Yildirir, Y.E., Improved direct and converse theorems in weighted Lorentz spaces, Bulletin of the Belgian Mathematical Society-Simon Stevin, 23 (2), 247-262, (2016).

[12] Yildirir, Y.E., and Doğu, A., Convolution and approximation in weighted Lorentz spaces, Journal of Mathematical Analysis, 7 (5), 54-60, (2016).

[13] Yildirir, Y.E., and Israfilov, D.M., The properties of convolution type operators in weighted Orlicz spaces, Glasnik matematički, 45 (2), 461-474, (2010).

[14] Doğu, A., Avşar, A. H., and Yildirir, Y.E., Some inequalities about convolution and trigonometric approximation in weighted Orlicz spaces, Proceeding of the Institute of Mathematics and Mechanics National Academy of Sciences of Azerbaijan, 44 (1), 107-115, (2018).

[15] Akgün, R., and Yildirir, Y.E., Convolution and Jackson inequalities in Musielak-Orlicz spaces, Turkish Journal of Mathematics, 42 (5), 2166-2185, (2018).

[16] Edwards, R. E., Fourier Series: A Modern Introduction Volume 2, 85, Springer Science \& Business Media, (2012). 